Abstract OP0226 - Table 1. Risk of hospitalised infection in Abatacept versus TNFi initiators: PS-matched analysis

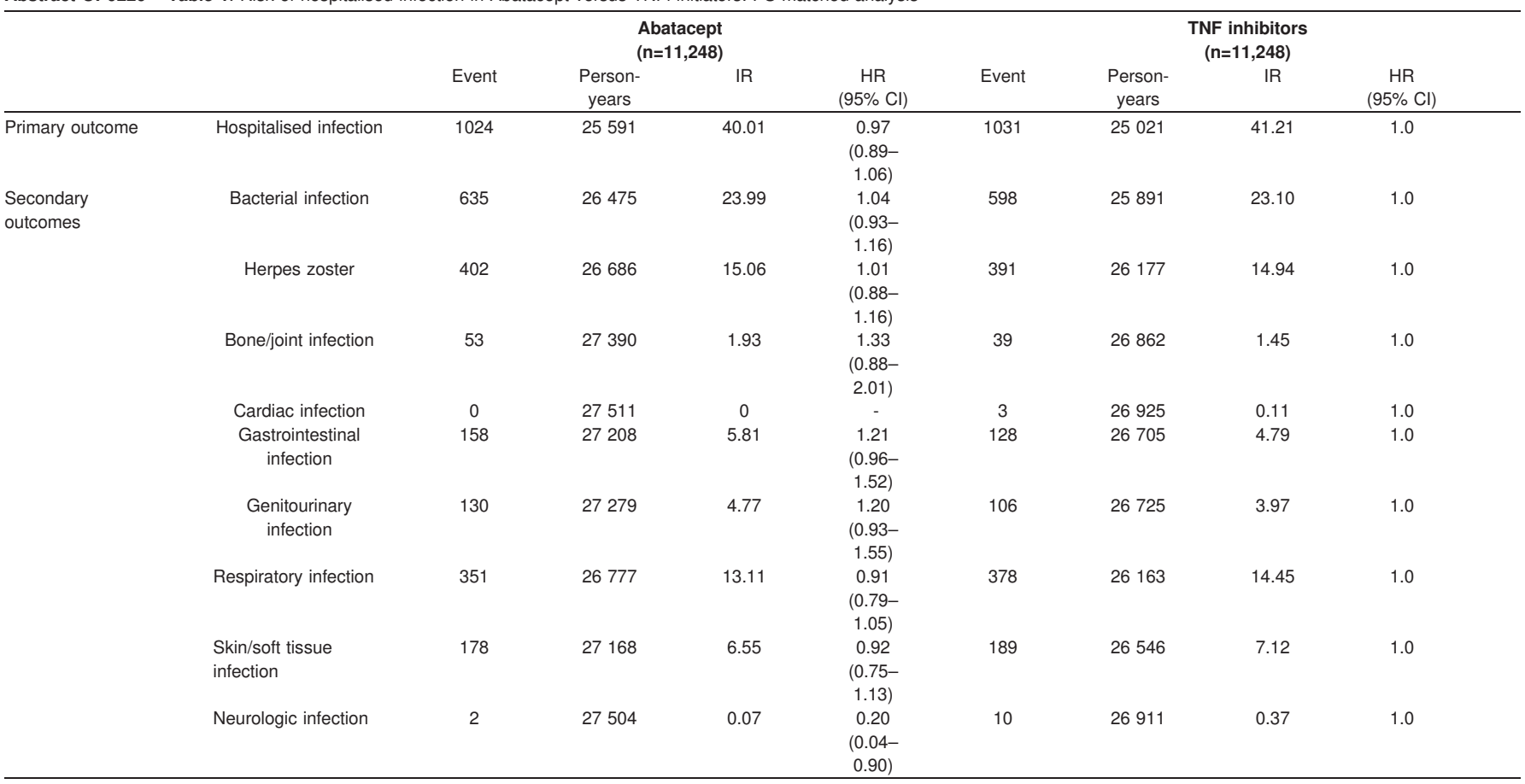

comparison of infection risk specific to different types of biologics used in a realworld setting.

Objectives: To evaluate the risk of hospitalised infection among RA patients who initiate abatacept versus TNFi.

Methods: We identified RA patients aged $\geq 18$ years with $\geq 2$ RA diagnoses separated by $7-365$ days using insurance claims data from Truven MarketScan database (2005-2015). New users of abatacept or TNFi (adalimumab, etanercept, certolizumab, golimumab, and infliximab) were included. To balance RA duration or severity between the groups, we excluded patients who previously used rituximab, tocilizumab or tofacitinib. We also excluded patients with malignancy, dialysis, HIV/AIDS, or organ transplantation. The primary outcome was a composite endpoint of hospitalised infection including bacterial, viral or opportunistic infection. Secondary outcomes were bacterial infection, herpes zoster, and infections affecting different organ systems. To control for over 50 baseline confounders, we performed 1:1 propensity score (PS) matching. We estimated incidence rate (IR) and hazard ratio $(\mathrm{HR})$ with $95 \%$ confidence interval $(\mathrm{Cl})$ of risk of hospitalised infection.

Results: We included 11,248 PS-matched pairs of abatacept and TNFi initiators with median age of 56 years. $83 \%$ were female. In the 1 year baseline period, $68 \%$ had any use of oral steroids and $55 \%$ used methotrexate. $18 \%$ had diabetes and $3 \%$ had hospitalised infection. Over the mean 2.3 year of followup, 1024 abatacept and 1,031 TNFi initiators were hospitalised for infection (table 1). The risk of any hospitalised infection was similar (HR $0.97,95 \% \mathrm{Cl}: 0.89$ to 1.06 ) between the two groups with the IR per 1000 person-years of 40.01 in abatacept and 41.21 in TNFi initiators. We found consistent results in the analyses of secondary outcomes. IR=events per 1000 person-years

Conclusions: In this large cohort of RA patients who used abatacept or TNFi as a first or second-line biologic agent, we found no difference in the risk of hospitalised infection between the two groups.

Acknowledgements: This study was funded by Bristol-Myers Squibb.

Disclosure of Interest: S. Chen: None declared, K. Liao: None declared, J. Liu: None declared, S. Kim Grant/research support from: Bristol-Myers Squibb, Roche, and Pfizer

DOI: 10.1136/annrheumdis-2018-eular.2003

\section{OP0227 \\ TIMING OF ABATACEPT BEFORE ELECTIVE ARTHROPLASTY AND POST-OPERATIVE OUTCOMES}

M. George ${ }^{1}$, J. Baker ${ }^{1}$, K. Winthrop ${ }^{2}$, E. Alemao ${ }^{3}$, L. Chen ${ }^{4}$, S. Connolly ${ }^{3}$, T. Simon ${ }^{3}$, Q. Wu ${ }^{1}$, F. Xie ${ }^{4}$, S. Yang ${ }^{4}$, J. Curtis ${ }^{4}{ }^{1}$ University of Pennsylvania, Philadelphia; ${ }^{2}$ Oregon Health and Science University, Portland; ${ }^{3}$ Bristol Myers Squibb, New York; ${ }^{4}$ University of Alabama at Birmingham, Birmingham, USA

Background: Guidelines recommend holding biologic DMARDs before major surgery, but few studies have examined perioperative timing of individual biologic therapies. ${ }^{1}$
Objectives: We aimed to determine whether holding abatacept infusions before elective hip or knee arthroplasty is associated with lower risk of adverse postoperative outcomes.

Methods: This retrospective cohort study using U.S. Medicare claims data from 2006-September 2015 evaluated adults with $\geq 2$ ICD9 codes for RA who received abatacept by infusion within 6 months of inpatient primary or revision total hip or knee arthroplasty. Infusions were selected as these procedures can be precisely dated in claims data. Patients with hip fracture, malignancy, pre-existing infection, non-elective procedures, or surgery after hospital day 3 were excluded. Logistic and Cox regression were used to assess associations between abatacept stop timing (time between most recent infusion and surgery in 4 week intervals based on dosing interval) and adverse outcomes: 1) hospitalised infection within 30 days (from discharge diagnoses, PPV $>80 \%$ ), 2) rate of prosthetic joint infection (PJI, ICD9 996.66) within 1 year, and 3) 30 day readmission (among patients with discharge to home, rehabilitation facility, or skilled nursing facility). Propensity scores based on the probability of being in each abatacept stop timing group were used to balance confounders across exposure groups using inverse probability weighting. Risk of hospitalised infection associated with methotrexate or with average glucocorticoid dose in the 3 months prior to surgery was assessed in abatacept treated patients using a reduced multivariable logistic regression model.

Results: Among 1537 surgeries in 1410 patients, there were $158(10.3 \%)$ hospitalised infections within 30 days (most commonly urinary, skin/soft tissue, and pneumonia), 34 (2.6/100 person-years) PJI within 1 year, and 108/1448 (7.5\%) 30 day readmissions. There were no significant differences in the rates of hospitalised infection, prosthetic joint infection, or 30 day readmission in patients who received abatacept within 4 weeks of surgery vs patients with longer stop timing (table 1). Among abatacept treated patients, glucocorticoid use (vs. none) was associated with a dose-dependent increase in the risk of hospitalised infection: $\leq 5$ $\mathrm{mg}$ [aOR 1.32 (0.88-1.98)], 5-10 mg [aOR 2.40 (1.54-3.73)],>10 mg [aOR 1.73 (0.74-4.06)]. Concomitant use of methotrexate was not associated with hospitalised infection risk [aOR $0.97(0.68-1.38)]$ (table 2).

Abstract OP0227 - Table 1 Association between abatacept stop timing and post-operative outcomes, from logistic and Cox regression with inverse probability weighting

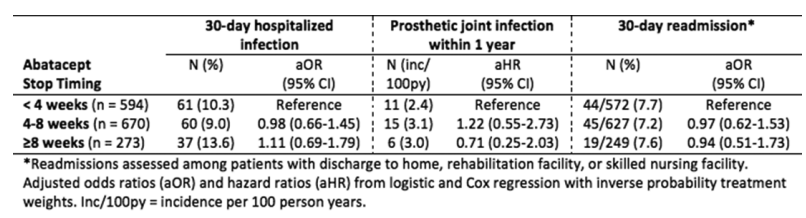


Abstract OP0227 - Table 2 Association between glucocorticoids and methotrexate with post-operative hospitalised infection among abatacept treated patients, logistic regression model

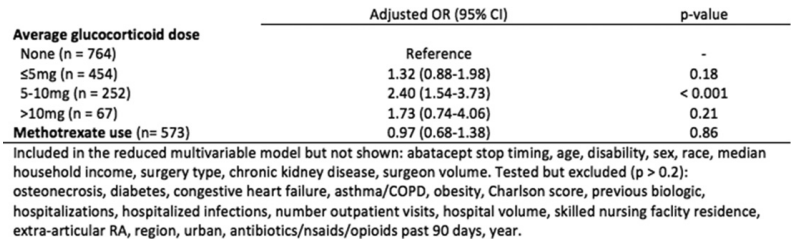

Conclusions: Holding intravenous abatacept for $\geq 4$ weeks (one dosing interval) was not associated with a lower risk of hospitalised infection, prosthetic joint infection, or 30 day readmission. Glucocorticoid use even at 5-10 $\mathrm{mg}$ per day was associated with significantly greater risk of post-operative infection.

\section{REFERENCE:}

[1] Goodman SM, et al. ACR/AAHKS Guideline for the Perioperative Management of Antirheumatic Medication in Patients With Rheumatic Diseases Undergoing Elective Total Hip or Total Knee Arthroplasty. Arthritis \& Rheum 2017;69:1538-1551.

Disclosure of Interest: M. George Grant/research support from: Bristol Myers Squibb, J. Baker: None declared, K. Winthrop Grant/research support from: Abbvie, Astellis, Galapagos, Eli Lilly, Pfizer, BMS, Roche, UCB, Consultant for: Abbvie, Astellis, Galapagos, Eli Lilly, Pfizer, BMS, Roche, UCB, E. Alemao Employee of: Bristol Myers Squibb, L. Chen: None declared, S. Connolly Employee of: Bristol Myers Squibb, T. Simon Employee of: Bristol Myers Squibb, Q. Wu: None declared, F. Xie: None declared, S. Yang: None declared, J. Curtis Grant/ research support from: Pfizer, Amgen, UCB, Myriad genetics, Bristol Myers Squibb, Consultant for: Pfizer, Amgen, UCB, Myriad genetics, Bristol Myers Squibb, Janssen

DOI: 10.1136/annrheumdis-2018-eular.3549

\section{OP0228 COMPARATIVE RISK OF BIOLOGIC THERAPIES AND RISK OF GLUCOCORTICOIDS IN PATIENTS WITH RHEUMATOID ARTHRITIS UNDERGOING ELECTIVE ARTHROPLASTY}

M. George ${ }^{1}$, J. Baker ${ }^{1}$, K. Winthrop ${ }^{2}$, E. Alemao ${ }^{3}$, L. Chen ${ }^{4}$, S. Connolly ${ }^{3}$, T. Simon ${ }^{3}$, Q. Wu ${ }^{1}$, F. Xie ${ }^{4}$, S. Yang ${ }^{4}$, J. Curtis ${ }^{4}$. ${ }^{1}$ University of Pennsylvania, Philadelphia; ${ }^{2}$ Oklahoma Health and Science University, Portland; ${ }^{3}$ Bristol Myers Squibb, New York; ${ }^{4}$ University of Alabama at Birmingham, Birmingham, USA

Background: Patients with RA undergoing major surgery are at high risk for infection. Different biologic DMARDs may be associated with different infection risks. $^{1}$

Objectives: Goals were 1) to compare post-operative infection risk after arthroplasty in patients with RA exposed to different biologics, and 2) examine associations between glucocorticoid use and post-operative infection.

Methods: A retrospective cohort study using U.S. Medicare data from 2006-September 2015 evaluated adults with $\geq 2$ ICD9 codes for RA undergoing elective inpatient primary or revision total knee or hip arthroplasty. Eligible patients received an infusion or prescription for abatacept, adalimumab, etanercept, infliximab, or tocilizumab within 8 weeks or a rituximab infusion within 16 weeks of surgery. Patients with hip fracture, malignancy, pre-existing infection, or non-elective surgery were excluded. Average glucocorticoid dose in the 3 months before surgery was calculated from oral prescriptions. Logistic or Cox regression evaluated associations between biologic exposure and post-operative outcomes: 1) hospitalised infection within 30 days (from discharge diagnoses, PPV $>80 \%$ ), 2) rate of prosthetic joint infection (PJI, ICD9 996.66) within 1 year, and 3) 30 day readmission among patients with discharge to home, skilled nursing facility, or inpatient rehab. Propensity scores based on the probability of receiving a specific biologic treatment were used to balance confounders across treatment groups using inverse probability weighting. Similar analyses were used to evaluate associations between glucocorticoid dose and outcomes in the same cohort, using inverse probability weighted analyses based on the probability of being in each glucocorticoid treatment category.

Results: Among 7929 surgeries in 7138 patients, there were 717 (9.0\%) hospitalised infections within 30 days of surgery (most commonly urinary, skin/soft tissue, and pneumonia), 192 (2.8/100 person-years) PJI within 1 year, and 465/7554 $(6.2 \%) 30$ day readmissions. There was no significant difference in the risk of hospitalised infection, PJI, or 30 day readmission across biologic treatment groups (table 1). Glucocorticoid dose $>10 \mathrm{mg} /$ day (mean $13.5 \pm 3.5 \mathrm{mg} /$ day) was associated with a significantly greater risk of hospitalised infection [aOR 2.37 (1.63-3.44)] and prosthetic joint infection [aHR 2.04 (1.09-3.84)] compared to no glucocorticoid use (table 1). Patients with glucocorticoid dose $>10 \mathrm{mg}$ also had a numerically greater risk of 30 day readmission that did not reach statistical significance [aOR 1.61 (0.99-2.61)] (table 1).

Abstract OP0228 - Table 1 Associations between preoperative biologic exposure or glucocorticoid dose and post-operative outcomes

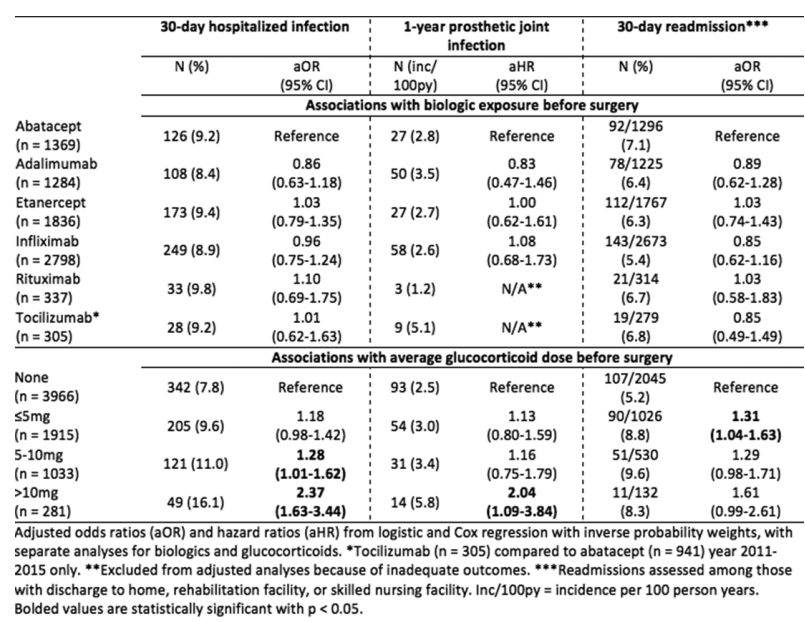

Conclusions: Risk of hospitalised infection, prosthetic joint infection, and read mission after arthroplasty was similar in patients with RA treated with different biologics. In contrast, glucocorticoid use, especially $>10 \mathrm{mg} /$ day, was associated with greater risk of hospitalised infection and PJI.

\section{REFERENCE:}

[1] Yun $\mathrm{H}$, et al. Comparative risk of hospitalized infection associated with biologic agents in rheumatoid arthritis patients enrolled in medicare. Arthritis \& Rheumatology 2016;68:56-66

Disclosure of Interest: M. George Grant/research support from: Bristol Myers Squibb, J. Baker: None declared, K. Winthrop Grant/research support from: Abbvie, Astellis, Galapagos, Eli Lilly, Pfizer, BMS, Roche, UCB, Consultant for: Abbvie, Astellis, Galapagos, Eli Lilly, Pfizer, BMS, Roche, UCB, E. Alemao Employee of: Bristol Myers Squibb, L. Chen: None declared, S. Connolly Employee of: Bristol Myers Squibb, T. Simon Employee of: Bristol Myers Squibb, Q. Wu: None declared, F. Xie: None declared, S. Yang: None declared, J. Curtis Grant research support from: Pfizer, Amgen, UCB, Myriad genetics, Bristol Myers Squibb, Consultant for: Pfizer, Amgen, UCB, Myriad genetics, Bristol Myers Squibb, Janssen

DOI: 10.1136/annrheumdis-2018-eular.1463

\section{OP0229 THE ASSOCIATION OF BIOLOGIC DRUG-LEVELS WITH INFECTION RISK: RESULTS FROM THE BRITISH SOCIETY FOR RHEUMATOLOGY BIOLOGICS REGISTER FOR RHEUMATOID ARTHRITIS}

M. Jani ${ }^{1}$, W.G. Dixon ${ }^{1}$, M. Lunt ${ }^{1}$, D. De Cock $^{1}$, J.D. Isaacs ${ }^{2}$, A.W. Morgan ${ }^{3}$, A. G. Wilson ${ }^{4}$, D. Plant ${ }^{5}, \mathrm{~K}$. Watson ${ }^{1}$, A. Barton ${ }^{5}, \mathrm{~K}$. Hyrich ${ }^{1,5}$, on behalf of BSRBR Control Centre Consortium. ${ }^{1}$ ARUK Centre for Epidemiology, University of Manchester, Manchester, ${ }^{2}$ University of Newcastle, Newcastle: ${ }^{3}$ University of Leeds, Leeds; ${ }^{4}$ University College of Dublin, Dublin; ${ }^{5}$ BRC, Manchester Foundation Trust, Manchester, UK

Background: High dose tumour necrosis factor inhibitor (TNFi) drugs are associated with an increased serious infection (SI) risk. ${ }^{1}$ It is feasible that high biologic levels predict dose-dependent adverse events such as SI. No registries have sys tematically evaluated the effect of drug levels on infection risk.

Objectives: To assess the effect of biologic drug levels in rheumatoid arthritis (RA) patients on (i) all infections (AI) (ii) SI (infections requiring hospitalisation, IV antibiotics or lead to death) 